\title{
Exploring Dynamics in the Far-Infrared with Terahertz Spectroscopy
}

\author{
Charles A. Schmuttenmaer* \\ Yale University, Department of Chemistry, 225 Prospect St., P. O. Box 208107 \\ New Haven, CT 06520-8107, USA
}

April, 2004

\section{Contents}

References with Titles

\section{References}

(1) Nuss, M. C.; Orenstein, J. In Millimeter and Submillimeter Wave Spectroscopy of Solids; Gruner, G., Ed.; Springer-Verlag: Berlin, 1998; Vol. 74.

(2) Mittleman, D. Sensing with Terahertz Radiation; Springer-Verlag: Berlin, 2003.

(3) Siegel, P. H., "Terahertz technology", IEEE Trans. Microw. Theory Tech. 2002, 50, 910.

(4) Beard, M. C.; Turner, G. M.; Schmuttenmaer, C. A., "Terahertz spectroscopy", J. Phys. Chem. B 2002, 106, 7146.

(5) Han, P. Y.; Zhang, X. C., "Free-space coherent broadband terahertz time-domain spectroscopy", Meas. Sci. Technol. 2001, 12, 1747.

(6) Ferguson, B.; Zhang, X. C., "Materials for terahertz science and technology", Nature Mater. 2002, 1, 26.

(7) Averitt, R. D.; Taylor, A. J., "Ultrafast optical and far-infrared quasiparticle dynamics in correlated electron materials", J. Phys.: Condens. Matter 2002, 14, R1357.

(8) Shan, J.; Nahata, A.; Heinz, T. F., "Terahertz time-domain spectroscopy based on nonlinear optics", J. Nonlinear Opt. Phys. Mater. 2002, 11, 31.

(9) Davies, A. G.; Linfield, E. H.; Johnston, M. B., "The development of terahertz sources and their applications", Phys. Med. Biol. 2002, 47,3679

(10) Kawase, K.; Shikata, J.; Ito, H., "Terahertz wave parametric source", J. Phys. D-Appl. Phys. 2002, 35, R1.

(11) Kimmitt, M. F., "Restrahlen to T-rays - 100 years of terahertz radiation", J. Biol. Phys. 2003, 29, 77.

(12) Special Issue: Proceedings of the First International Conference on Biomedical Imaging and Sensing Applications of $\mathrm{THz}$ Technology Phys. Med. Biol., 2002; Vol. 47.

(13) J. Biol. Phys., 2003; Vol. 29.

(14) Smith, P. R.; Auston, D. H.; Nuss, M. C., "Subpicosecond photoconducting dipole antennas", IEEE J. Quantum Electron. 1988, 24, 255.

(15) Fattinger, C.; Grischkowsky, D., "Terahertz Beams", Appl. Phys. Lett. 1989, 54, 490.

(16) van Exter, M.; Fattinger, C.; Grischkowsky, D., "High-Brightness Terahertz Beams Characterized with an Ultrafast Detector", Appl. Phys. Lett. 1989, 55, 337.

(17) van Exter, M.; Grischkowsky, D. R., "Characterization of an Optoelectronic Terahertz Beam System", IEEE Trans. Microw. Theory Tech. 1990, 38, 1684.
(18) Ralph, S. E.; Grischkowsky, D., "Trap-Enhanced Electric-Fields in Semi-Insulators - the Role of Electrical and Optical Carrier Injection", Appl. Phys. Lett. 1991, 59, 1972.

(19) Katzenellenbogen, N.; Grischkowsky, D., "Efficient Generation of 380 Fs Pulses of Thz Radiation by Ultrafast Laser-Pulse Excitation of a Biased Metal- Semiconductor Interface", Appl. Phys. Lett. 1991, 58, 222.

(20) Warren, A. C.; Katzenellenbogen, N.; Grischkowsky, D.; Woodall, J. M.; Melloch, M. R.; Otsuka, N., "Subpicosecond, Freely Propagating Electromagnetic Pulse Generation and Detection Using Gaas-as Epilayers", Appl. Phys. Lett. 1991, 58, 1512.

(21) Ludwig, C.; Kuhl, J., "Studies of the temporal and spectral shape of terahertz pulses generated from photoconducting switches", Appl. Phys. Lett. 1996, 69, 1194.

(22) Heiliger, H. M.; Vosseburger, M.; Roskos, H. G.; Kurz, H.; Hey, R.; Ploog, K., "Application of liftoff low-temperature-grown GaAs on transparent substrates for THz signal generation", Appl. Phys. Lett. 1996, 69, 2903.

(23) Tani, M.; Matsuura, S.; Sakai, K.; Nakashima, S., "Emission characteristics of photoconductive antennas based on low-temperaturegrown GaAs and semi-insulating GaAs", Appl. Optics 1997, 36, 7853.

(24) Lin, P. I.; Chen, S. F.; Wu, K. H.; Juang, J. Y.; Uhn, T. M.; Gou, Y. S., "Characteristics of photogenerated bipolar terahertz radiation in biased photoconductive switches", Jpn. J. Appl. Phys. Part 2 - Lett. 2002, 41, L1158.

(25) Liu, T. A.; Tani, M.; Pan, C. L., "THz radiation emission properties of multienergy arsenic-ion- implanted GaAs and semi-insulating GaAs based photoconductive antennas", J. Appl. Phys. 2003, 93, 2996.

(26) Pedersen, J. E.; Keiding, S. R.; Sorensen, C. B.; Lindelof, P. E.; Ruhle, W. W.; Zhou, X. Q., "5-Thz Bandwidth from a Gaas-on-Silicon Photoconductive Receiver", J. Appl. Phys. 1993, 74, 7022.

(27) Zhang, J. Z.; Chen, Z. Z.; Chen, X. M., "FDTD analysis of photoconducting antennas for millimeter-wave generation", Int. J. RF Microw. Comput-Aid. Eng. 2000, 10, 213.

(28) Chung, Y. S.; Cheon, C.; Son, J. H.; Hahn, S. Y., "FDTD analysis of propagation characteristics of terahertz electromagnetic pulses", IEEE Trans. Magn. 2000, 36, 951.

(29) Jepsen, P. U.; Jacobsen, R. H.; Keiding, S. R., "Generation and detection of terahertz pulses from biased semiconductor antennas", J. Opt. Soc. Am. B-Opt. Phys. 1996, 13, 2424.

(30) Duvillaret, L.; Garet, F.; Roux, J. F.; Coutaz, J. L., "Analytical modeling and optimization of terahertz time-domain spectroscopy experiments using photoswitches as antennas", IEEE J. Sel. Top. Quantum Electron. 2001, 7, 615 . 
(31) Kindt, J. T.; Schmuttenmaer, C. A., "Far-infrared dielectric properties of polar liquids probed by femtosecond terahertz pulse spectroscopy", J. Phys. Chem. 1996, 100, 10373.

(32) Melloch, M. R.; Woodall, J. M.; Harmon, E. S.; Otsuka, N.; Pollak, F. H.; Nolte, D. D.; Feenstra, R. M.; Lutz, M. A., "LowTemperature-Grown Iii-V Materials", Ann. Rev. Mater. Sci. 1995, $25,547$.

(33) Wang, H. H.; Whitaker, J. F.; Chin, A.; Mazurowski, J.; Ballingall, J. M., "Subpicosecond Carrier Response of Unannealed LowTemperature-Grown Gaas Vs Temperature", J. Electron. Mater. 1993, 22, 1461.

(34) Beard, M. C.; Turner, G. M.; Schmuttenmaer, C. A., "Subpicosecond carrier dynamics in low-temperature grown GaAs as measured by time-resolved terahertz spectroscopy", J. Appl. Phys. 2001, 90, 5915.

(35) Tonouchi, M.; Tani, M.; Wang, Z.; Sakai, K.; Hangyo, M.; Wada N.; Murakami, Y., "Enhanced THz radiation from YBCO thin film bow-tie antennas with hyper-hemispherical $\mathrm{MgO}$ lens", IEEE Trans. Appl. Supercond. 1997, 7, 2913.

(36) Tani, M.; Tonouchi, M.; Hangyo, M.; Wang, Z.; Onodera, N.; Sakai, K., "Emission properties of YBa2Cu3O7-delta-film photoswitches as Terahertz radiation sources", Jpn. J. Appl. Phys. Part 1 - Regul. Pap. Short Notes Rev. Pap. 1997, 36, 1984.

(37) Tanichi, N.; Wada, N.; Nagashima, T.; Tonouchi, M.; Hangyo, M.; Tani, M.; Sakai, K., "Terahertz radiation from a-axis oriented YBCO thin films", Physica C 1997, 293, 229.

(38) Shikii, S.; Tanichi, N.; Nagashima, T.; Tonouchi, M.; Hangyo, M.; Tani, M.; Sakai, K., "Enhanced THz radiation from YBCO using aaxis oriented thin films excited by ultrashort optical pulses", IEICE Trans. Electron. 1997, E80C, 1297.

(39) Pedersen, J. E.; Balslev, I.; Hvam, J. M.; Keiding, S. R., "Terahertz Pulses from Semiconductor-Air Interfaces", Appl. Phys. Lett. 1992, $61,1372$.

(40) Andrews, S. R.; Armitage, A.; Huggard, P. G.; Hussain, A., "Optimization of photoconducting receivers for $\mathrm{THz}$ spectroscopy", Phys. Med. Biol. 2002, 47, 3705.

(41) Xu, L.; Zhang, X. C.; Auston, D. H.; Jalali, B., "Terahertz Radiation from Large Aperture Si P-I-N-Diodes", Appl. Phys. Lett. $1991,59,3357$.

(42) Zhang, X. C.; Auston, D. H., "Optically Induced Thz Electromagnetic-Radiation from Planar Photoconducting Structures", J. Electromagn. Waves Appl. 1992, 6, 85.

(43) Hu, B. B.; Weling, A. S.; Auston, D. H.; Kuznetsov, A. V.; Stanton, C. J., "Dc-Electric-Field Dependence of Thz RadiationInduced by Femtosecond Optical-Excitation of Bulk Gaas", Phys. Rev. B 1994, 49, 2234.

(44) Leitenstorfer, A.; Hunsche, S.; Shah, J.; Nuss, M. C.; Knox, W. H., "Detectors and sources for ultrabroadband electro-optic sampling: Experiment and theory", Appl. Phys. Lett. 1999, 74, 1516.

(45) Zhang, X. C.; Darrow, J. T.; Hu, B. B.; Auston, D. H.; Schmidt, M. T.; Tham, P.; Yang, E. S., "Optically Induced ElectromagneticRadiation from Semiconductor Surfaces", Appl. Phys. Lett. 1990, 56,2228

(46) Jin, Y.; Ma, X. F.; Wagoner, G. A.; Alexander, M.; Zhang, X. C., "Anomalous Optically Generated Thz Beams from Metal Gaas Interfaces", Appl. Phys. Lett. 1994, 65, 682.

(47) Zhang, X. C.; Hu, B. B.; Darrow, J. T.; Auston, D. H., "Generation of Femtosecond Electromagnetic Pulses from Semiconductor Surfaces", Appl. Phys. Lett. 1990, 56, 1011.

(48) Greene, B. I.; Saeta, P. N.; Dykaar, D. R.; Schmittrink, S.; Chuang, S. L., "Far-Infrared Light Generation at Semiconductor Surfaces and Its Spectroscopic Applications", IEEE J. Quantum Electron. 1992, 28, 2302.

(49) Li, M.; Sun, F. G.; Wagoner, G. A.; Alexander, M.; Zhang, X. C., "Measurement and Analysis of Terahertz Radiation from Bulk Semiconductors", Appl. Phys. Lett. 1995, 67, 25.

(50) Kondo, T.; Sakamoto, M.; Tonouchi, M.; Hangyo, M., "Terahertz radiation from (111) InAs surface using $1.55 \mathrm{mu} \mathrm{m}$ femtosecond laser pulses", Jpn. J. Appl. Phys. Part 2 - Lett. 1999, 38, L1035.
(51) Han, P. Y.; Huang, X. G.; Zhang, X. C., "Direct characterization of terahertz radiation from the dynamics of the semiconductor surface field", Appl. Phys. Lett. 2000, 77, 2864.

(52) Kono, S.; Gu, P.; Tani, M.; Sakai, K., "Temperature dependence of terahertz radiation from n-type InSb and n-type InAs surfaces", Appl. Phys. B-Lasers Opt. 2000, 71, 901.

(53) Gu, P.; Tani, M.; Kono, S.; Sakai, K.; Zhang, X. C., "Study of terahertz radiation from InAs and InSb", J. Appl. Phys. 2002, 91, 5533.

(54) Malevich, V. L., "Monte Carlo simulation of THz-pulse generation from semiconductor surface", Semicond. Sci. Technol. 2002, 17, 551.

(55) Nakajima, M.; Takahashi, M.; Hangyo, M., "Strong enhancement of THz radiation intensity from semi- insulating GaAs surfaces at high temperatures", Appl. Phys. Lett. 2002, 81, 1462.

(56) Zhang, X. C.; Jin, Y.; Hewitt, T. D.; Sangsiri, T.; Kingsley, L. E.; Weiner, M., "Magnetic Switching of Thz Beams", Appl. Phys. Lett. 1993, 62, 2003.

(57) Sarukura, N.; Ohtake, H.; Izumida, S.; Liu, Z. L., "High average-power $\mathrm{THz}$ radiation from femtosecond laser- irradiated InAs in a magnetic field and its elliptical polarization characteristics", J. Appl. Phys. 1998, 84,654 .

(58) Izumida, S.; Ono, S.; Liu, Z. L.; Ohtake, H.; Sarukura, N., "Spectrum control of THz radiation from InAs in a magnetic field by duration and frequency chirp of the excitation pulses", Appl. Phys. Lett. 1999, 75, 451.

(59) Ono, S.; Tsukamoto, T.; Sakai, M.; Liu, Z. L.; Ohtake, H.; Sarukura, N.; Nishizawa, S.; Nakanishi, A.; Yoshida, M., "Compact THz-radiation source consisting of a bulk semiconductor, a mode-locked fiber laser, and a 2 T permanent magnet", Rev. Sci. Instrum. 2000, 71, 554.

(60) McLaughlin, R.; Chen, Q.; Corchia, A.; Ciesla, C. M.; Arnone, D. D.; Zhang, X. C.; Jones, G. A. C.; Lindfield, E. H.; Pepper, M., "Enhanced coherent terahertz emission from indium arsenide", J. Mod. Opt. 2000, 47,1847

(61) Ohtake, H.; Ono, S.; Sakai, M.; Liu, Z. L.; Tsukamoto, T.; Sarukura, N., "Saturation of THz-radiation power from femtosecond-laser- irradiated InAs in a high magnetic field", Appl. Phys. Lett. 2000, 76, 1398.

(62) Ohtake, H.; Ono, S.; Sakai, M.; Liu, Z.; Sarukura, N., "Intense THz radiation from femtosecond laser pulses irradiated InAs in a strong magnetic field", J. Lumines. 2000, 87-9, 902.

(63) Liu, Z. L.; Ohtake, H.; Izumida, S.; Ono, S.; Sarukura, N., "Intense THzradiation from semiconductors under magnetic field irradiated with femtosecond laser pulses", Opt. Quantum Electron. 2000, 32, 521.

(64) Heyman, J. N.; Neocleous, P.; Hebert, D.; Crowell, P. A.; Muller, T.; Unterrainer, K., "Terahertz emission from GaAs and InAs in a magnetic field", Phys. Rev. B 2001, 64, 085202.

(65) Ono, S.; Tsukamoto, T.; Kawahata, E.; Yano, T.; Ohtake, H.; Sarukura, $\mathrm{N}$. , "Terahertz radiation from a shallow incidence-angle InAs emitter in a magnetic field irradiated with femtosecond laser pulses", Appl. Optics 2001, 40, 1369.

(66) Migita, M.; Hangyo, M., "Pump-power dependence of $\mathrm{THz}$ radiation from InAs surfaces under magnetic fields excited by ultrashort laser pulses", Appl. Phys. Lett. 2001, 79, 3437.

(67) Ohtake, H.; Suzuki, Y.; Sarukura, N.; Ono, S.; Tsukamoto, T.; Nakanishi, A.; Nishizawa, S.; Stock, M. L.; Yoshida, M.; Endert, H., "THz-radiation emitter and receiver system based on a $2 \mathrm{~T}$ permanent magnet, $1040 \mathrm{~nm}$ compact fiber laser and pyroelectric thermal receiver", Jpn. J. Appl. Phys. Part 2 - Lett. 2001, 40, L1223.

(68) Hangyo, M.; Migita, M.; Nakayama, K., "Magnetic field and temperature dependence of terahertz radiation from InAs surfaces excited by femtosecond laser pulses", J. Appl. Phys. 2001, 90, 3409.

(69) Ohtake, H.; Murakami, H.; Yano, T.; Ono, S.; Sarukura, N.; Takahashi, H.; Suzuki, Y.; Nishijima, G.; Watanabe, K., "Anomalous power and spectrum dependence of terahertz radiation from femtosecond-laserirradiated indium arsenide in high magnetic fields up to 14 T", Appl. Phys. Lett. 2003, 82, 1164.

(70) Weiss, C.; Wallenstein, R.; Beigang, R., "Magnetic-field-enhanced generation of terahertz radiation in semiconductor surfaces", Appl. Phys. Lett. 2000, 77, 4160.

(71) Johnston, M. B.; Corchia, A.; Dowd, A.; Linfield, E. H.; Davies, A. G.; McLaughlin, R.; Arnone, D. D.; Pepper, M., "Magnetic-field-induced 
enhancement of terahertz emission from III-V semiconductor surfaces", Physica E 2002, 13, 896.

(72) Takahashi, H.; Suzuki, Y.; Sakai, M.; Ono, S.; Sarukura, N.; Sugiura, T.; Hirosumi, T.; Yoshida, M., "Significant enhancement of terahertz radiation from InSb by use of a compact fiber laser and an external magnetic field", Appl. Phys. Lett. 2003, 82, 2005.

(73) Shan, J.; Weiss, C.; Wallenstein, R.; Beigang, R.; Heinz, T. F., "Origin of magnetic field enhancement in the generation of terahertz radiation from semiconductor surfaces", Opt. Lett. 2001, 26,849 .

(74) Johnston, M. B.; Whittaker, D. M.; Corchia, A.; Davies, A. G.; Linfield, E. H., "Theory of magnetic-field enhancement of surfacefield terahertz emission", J. Appl. Phys. 2002, 91, 2104.

(75) Johnston, M. B.; Whittaker, D. M.; Corchia, A.; Davies, A. G.; Linfield, E. H., "Simulation of terahertz generation at semiconductor surfaces", Phys. Rev. B 2002, 65, 165301.

(76) Andrews, S. R.; Armitage, A.; Huggard, P. G.; Shaw, C. J.; Moore, G. P.; Grey, R., "Magnetic field dependence of terahertz emission from an optically excited GaAs p-i-n diode", Phys. Rev. B 2002, 66, 085307.

(77) Sarukura, N.; Liu, Z.; Ohtake, H.; Izumida, S.; Yamanaka, T.; Segawa, Y.; Itatani, T.; Sugaya, T.; Nakagawa, T.; Sugiyama, Y., "All-solid-state, $\mathrm{THz}$ radiation source using a saturable Bragg reflector in a femtosecond mode-locked laser", Jpn. J. Appl. Phys. Part 2 - Lett. 1997, 36, L560.

(78) Sarukura, N.; Ohtake, H.; Liu, Z.; Itatani, T.; Sugaya, T.; Nakagawa, T.; Sugiyama, Y., "THz-radiation generation from an intracavity saturable Bragg reflector in a magnetic field", Jpn. J. Appl. Phys. Part 2 - Lett. 1998, 37, L125.

(79) Liu, T. A.; Huang, K. F.; Pan, C. L.; Liu, Z. L.; Ono, S.; Ohtake, H.; Sarukura, N., "THz radiation from intracavity saturable Bragg reflector in magnetic field with self-started mode-locking by strained saturable Bragg reflector", Jpn. J. Appl. Phys. Part 2 Lett. 1999, 38, L1333.

(80) Liu, Z. L.; Ono, S.; Ohtake, H.; Sarukura, N.; Liu, T. A.; Huang, K. F.; Pan, C. L., "Efficient terahertz radiation generation from a bulk InAs mirror as an intracavity terahertz radiation emitter", Jpn. $J$. Appl. Phys. Part 2 - Lett. 2000, 39, L366.

(81) Darrow, J. T.; Hu, B. B.; Zhang, X. C.; Auston, D. H., "Subpicosecond Electromagnetic Pulses from Large-Aperture Photoconducting Antennas", Opt. Lett. 1990, 15, 323.

(82) Darrow, J. T.; Zhang, X. C.; Auston, D. H., "Power Scaling of Large-Aperture Photoconducting Antennas", Appl. Phys. Lett. 1991, 58, 25 .

(83) Benicewicz, P. K.; Roberts, J. P.; Taylor, A. J., "Scaling of Terahertz Radiation from Large-Aperture Biased Photoconductors", J. Opt. Soc. Am. B-Opt. Phys. 1994, 11, 2533.

(84) You, D.; Jones, R. R.; Bucksbaum, P. H.; Dykaar, D. R., "Generation of High-Power Sub-Single-Cycle 500-Fs Electromagnetic Pulses", Opt. Lett. 1993, 18, 290.

(85) Hattori, T.; Tukamoto, K.; Nakatsuka, H., "Time-resolved study of intense terahertz pulses generated by a large-aperture photoconductive antenna", Jpn. J. Appl. Phys. Part 1 - Regul. Pap. Short Notes Rev. Pap. 2001, 40, 4907.

(86) Zhao, G.; Schouten, R. N.; van der Valk, N.; Wenckebach, W. T.; Planken, P. C. M., "Design and performance of a THz emission and detection setup based on a semi-insulating GaAs emitter", Rev. Sci. Instrum. 2002, 73, 1715.

(87) Auston, D. H.; Nuss, M. C., "Electrooptic Generation and Detection of Femtosecond Electrical Transients", IEEE J. Quantum Electron. 1988, 24, 184.

(88) Carrig, T. J.; Rodriguez, G.; Clement, T. S.; Taylor, A. J.; Stewart, K. R., "Scaling of Terahertz Radiation Via Optical Rectification in Electrooptic Crystals", Appl. Phys. Lett. 1995, 66, 121.

(89) Chen, Q.; Zhang, X. C., "Polarization modulation in optoelectronic generation and detection of terahertz beams", Appl. Phys. Lett. 1999, 74, 3435 .
(90) Hu, B. B.; Zhang, X. C.; Auston, D. H.; Smith, P. R., "Free-Space Radiation from Electrooptic Crystals", Appl. Phys. Lett. 1990, 56, 506.

(91) Nahata, A.; Weling, A. S.; Heinz, T. F., "A wideband coherent terahertz spectroscopy system using optical rectification and electro-optic sampling", Appl. Phys. Lett. 1996, 69, 2321.

(92) Rice, A.; Jin, Y.; Ma, X. F.; Zhang, X. C.; Bliss, D.; Larkin, J.; Alexander, M., "Terahertz optical rectification from (110) zincblende crystals", Appl. Phys. Lett. 1994, 64, 1324.

(93) Xu, L.; Zhang, X. C.; Auston, D. H., "Terahertz Beam Generation by Femtosecond Optical Pulses in Electrooptic Materials", Appl. Phys. Lett. 1992, 61, 1784

(94) Zhang, X. C.; Jin, Y.; Ma, X. F., "Coherent Measurement of Thz Optical Rectification from Electrooptic Crystals", Appl. Phys. Lett. 1992, 61, 2764.

(95) Han, P. Y.; Zhang, X. C., "Coherent, broadband midinfrared terahertz beam sensors", Appl. Phys. Lett. 1998, 73, 3049.

(96) Beard, M. C.; Turner, G. M.; Schmuttenmaer, C. A., "Transient photoconductivity in GaAs as measured by time- resolved terahertz spectroscopy", Phys. Rev. B 2000, 62, 15764.

(97) Reimann, K.; Smith, R. P.; Weiner, A. M.; Elsaesser, T.; Woerner, M., "Direct field-resolved detection of terahertz transients with amplitudes of megavolts per centimeter", Opt. Lett. 2003, 28, 471.

(98) Han, P. Y.; Cho, G. C.; Zhang, X. C., "Broad band mid-infrared THz pulse: Measurement technique and applications", J. Nonlinear Opt. Phys. Mater. 1999, 8, 89 .

(99) Huber, R.; Brodschelm, A.; Tauser, F.; Leitenstorfer, A., "Generation and field-resolved detection of femtosecond electromagnetic pulses tunable up to $41 \mathrm{THz}$, Appl. Phys. Lett. 2000, 76, 3191.

(100) Sinyukov, A. M.; Hayden, L. M., "Generation and detection of terahertz radiation with multilayered electro-optic polymer films", Opt. Lett. 2002, $27,55$.

(101) Han, P. Y.; Tani, M.; Pan, F.; Zhang, X. C., "Use of the organic crystal DAST for terahertz beam applications", Opt. Lett. 2000, 25, 675.

(102) Carey, J. J.; Bailey, R. T.; Pugh, D.; Sherwood, J. N.; Cruickshank, F. R.; Wynne, K., "Terahertz pulse generation in an organic crystal by optical rectification and resonant excitation of molecular charge transfer", Appl. Phys. Lett. 2002, 81, 4335.

(103) Jiang, Z. P.; Zhang, X. C., "Terahertz imaging via electrooptic effect", IEEE Trans. Microw. Theory Tech. 1999, 47, 2644.

(104) Wu, Q.; Hewitt, T. D.; Zhang, X. C., "Two-dimensional electro-optic imaging of THz beams", Appl. Phys. Lett. 1996, 69, 1026.

(105) Wu, Q.; Zhang, X. C., "Free-space electro-optics sampling of midinfrared pulses", Appl. Phys. Lett. 1997, 71, 1285.

(106) Wu, Q.; Litz, M.; Zhang, X. C., "Broadband detection capability of ZnTe electro-optic field detectors", Appl. Phys. Lett. 1996, 68, 2924.

(107) Wu, Q.; Zhang, X. C., "Ultrafast electro-optic field sensors", Appl. Phys. Lett. 1996, 68, 1604.

(108) Bakker, H. J.; Cho, G. C.; Kurz, H.; Wu, Q.; Zhang, X. C., "Distortion of terahertz pulses in electro-optic sampling", J. Opt. Soc. Am. B-Opt. Phys. 1998, 15, 1795.

(109) Gallot, G.; Grischkowsky, D., "Electro-optic detection of terahertz radiation", J. Opt. Soc. Am. B-Opt. Phys. 1999, 16, 1204.

(110) Planken, P. C. M.; Nienhuys, H. K.; Bakker, H. J.; Wenckebach, T., "Measurement and calculation of the orientation dependence of terahertz pulse detection in ZnTe", J. Opt. Soc. Am. B-Opt. Phys. 2001, 18, 313.

(111) Vosseburger, M.; Brucherseifer, M.; Cho, G. C.; Roskos, H. G.; Kurz, H., "Propagation effects in electro-optic sampling of terahertz pulses in GaAs", Appl. Optics 1998, 37, 3368.

(112) Jiang, Z. P.; Zhang, X. C., "Single-shot spatiotemporal terahertz field imaging", Opt. Lett. 1998, 23, 1114.

(113) Jiang, Z. P.; Zhang, X. C., "Electro-optic measurement of THz field pulses with a chirped optical beam", Appl. Phys. Lett. 1998, 72, 1945.

(114) Jiang, Z. P.; Zhang, X. C., "Measurement of spatio-temporal terahertz field distribution by using chirped pulse technology", IEEE J. Quantum Electron. 2000, 36, 1214.

(115) Sun, F. G.; Jiang, Z. P.; Zhang, X. C., "Analysis of terahertz pulse measurement with a chirped probe beam", Appl. Phys. Lett. 1998, 73, 2233. 
(116) Shan, J.; Weling, A. S.; Knoesel, E.; Bartels, L.; Bonn, M.; Nahata, A.; Reider, G. A.; Heinz, T. F., "Single-shot measurement of terahertz electromagnetic pulses by use of electro-optic sampling", Opt. Lett. 2000, 25, 426.

(117) Kaindl, R. A.; Eickemeyer, F.; Woerner, M.; Elsaesser, T., "Broadband phase-matched difference frequency mixing of femtosecond pulses in GaSe: Experiment and theory", Appl. Phys. Lett. 1999, 75, 1060.

(118) Bonvalet, A.; Joffre, M.; Martin, J. L.; Migus, A., "Generation of Ultrabroadband Femtosecond Pulses in the Midinfrared by Optical Rectification of 15 Fs Light-Pulses at $100 \mathrm{Mhz}$ Repetition Rate", Appl. Phys. Lett. 1995, 67, 2907.

(119) Kono, S.; Tani, M.; Sakai, K., "Coherent detection of mid-infrared radiation up to $60 \mathrm{THz}$ with an LT-GaAs photoconductive antenna", IEE Proc.-Optoelectron. 2002, 149, 105.

(120) Kono, S.; Tani, M.; Sakai, K., "Ultrabroadband photoconductive detection: Comparison with free- space electro-optic sampling", Appl. Phys. Lett. 2001, 79, 898.

(121) Park, S. G.; Melloch, M. R.; Weiner, A. M., "Comparison of terahertz waveforms measured by electro-optic and photoconductive sampling", Appl. Phys. Lett. 1998, 73, 3184.

(122) Park, S. G.; Melloch, M. R.; Weiner, A. M., "Analysis of terahertz waveforms measured by photoconductive and electrooptic sampling", IEEE J. Quantum Electron. 1999, 35, 810.

(123) Kawase, K.; Sato, M.; Taniuchi, T.; Ito, H., "Coherent tunable THz-wave generation from $\mathrm{LiNbO3}$ with monolithic grating coupler", Appl. Phys. Lett. 1996, 68, 2483.

(124) Kawase, K.; Sato, M.; Nakamura, K.; Taniuchi, T.; Ito, H., "Unidirectional radiation of widely tunable $\mathrm{THz}$ wave using a prism coupler under noncollinear phase matching condition", Appl. Phys. Lett. 1997, 71, 753.

(125) Kawase, K.; Shikata, J.; Sato, M.; Taniuchi, T.; Ito, H., "Widely tunable coherent terahertz wave generation using nonlinear optical effect", Electron. Commun. Jpn. Pt. II-Electron. 1998, 81, 10.

(126) Ito, H.; Kawase, K.; Shikata, J., "Widely tunable THz-wave generation by nonlinear optics", IEICE Trans. Electron. 1998, E81C, 264

(127) Shikata, J.; Sato, M.; Taniuchi, T.; Ito, H., "Enhancement of terahertz-wave output from $\mathrm{LiNbO3}$ optical parametric oscillators by cryogenic cooling", Opt. Lett. 1999, 24, 202.

(128) Shikata, J.; Kawase, K.; Karino, K.; Taniuchi, T.; Ito, H., "Tunable terahertz-wave parametric oscillators using $\mathrm{LiNbO3}$ and $\mathrm{MgO}$ : LiNbO3 crystals", IEEE Trans. Microw. Theory Tech. 2000, 48, 653.

(129) Sato, A.; Kawase, K.; Minamide, H.; Wada, S.; Ito, H., "Tabletop terahertz-wave parametric generator using a compact, diodepumped Nd : YAG laser", Rev. Sci. Instrum. 2001, 72, 3501.

(130) Lee, Y. S.; Norris, T. B., "Terahertz pulse shaping and optimal waveform generation in poled ferroelectric crystals", J. Opt. Soc. Am. B-Opt. Phys. 2002, 19, 2791.

(131) Kawase, K.; Hatanaka, T.; Takahashi, H.; Nakamura, K.; Taniuchi, T.; Ito, H., "Tunable terahertz-wave generation from DAST crystal by dual signal-wave parametric oscillation of periodically poled lithium niobate", Opt. Lett. 2000, 25, 1714.

(132) Weiss, C.; Torosyan, G.; Avetisyan, Y.; Beigang, R., "Generation of tunable narrow-band surface-emitted terahertz radiation in periodically poled lithium niobate", Opt. Lett. 2001, 26, 563.

(133) Weiss, C.; Torosyan, G.; Meyn, J. P.; Wallenstein, R.; Beigang, R.; Avetisyan, Y., "Tuning characteristics of narrowband $\mathrm{THz}$ radiation generated via optical rectification in periodically poled lithium niobate", Opt. Express 2001, 8, 497.

(134) Hamster, H.; Sullivan, A.; Gordon, S.; White, W.; Falcone, R. W., "Subpicosecond, Electromagnetic Pulses from Intense LaserPlasma Interaction", Phys. Rev. Lett. 1993, 71, 2725.

(135) Hamster, H.; Sullivan, A.; Gordon, S.; Falcone, R. W., "ShortPulse Terahertz Radiation from High-Intensity-Laser- Produced Plasmas", Phys. Rev. E 1994, 49, 671.
(136) Loffler, T.; Jacob, F.; Roskos, H. G., "Generation of terahertz pulses by photoionization of electrically biased air", Appl. Phys. Lett. 2000, 77, 453.

(137) Cook, D. J.; Hochstrasser, R. M., "Intense terahertz pulses by four-wave rectification in air", Opt. Lett. 2000, 25, 1210.

(138) Chen, Q.; Jiang, Z. P.; Tani, M.; Zhang, X. C., "Electro-optic terahertz transceiver", Electron. Lett. 2000, 36, 1298.

(139) Chen, Q.; Tani, M.; Jiang, Z. P.; Zhang, X. C., "Electro-optic transceivers for terahertz-wave applications", J. Opt. Soc. Am. B-Opt. Phys. 2001, 18, 823.

(140) Tani, M.; Jiang, Z. P.; Zhang, X. C., "Photoconductive terahertz transceiver", Electron. Lett. 2000, 36, 804.

(141) Greene, B. I.; Federici, J. F.; Dykaar, D. R.; Jones, R. R.; Bucksbaum, P. H., "Interferometric Characterization of 160 Fs Far-Infrared LightPulses", Appl. Phys. Lett. 1991, 59, 893.

(142) Prabhu, S. S.; Ralph, S. E.; Melloch, M. R.; Harmon, E. S., "Carrier dynamics of low-temperature-grown $\mathrm{GaAs}$ observed via $\mathrm{THz}$ spectroscopy", Appl. Phys. Lett. 1997, 70, 2419.

(143) Ralph, S. E.; Chen, Y.; Woodall, J.; McInturff, D., "Subpicosecond photoconductivity of In $0.53 \mathrm{Ga} 0.47 \mathrm{As}$ : Intervalley scattering rates observed via THz spectroscopy", Phys. Rev. B 1996, 54, 5568.

(144) Nahata, A.; Heinz, T. F., "Detection of freely propagating terahertz radiation by use of optical second-harmonic generation", Opt. Lett. 1998, $23,67$.

(145) Bromage, J.; Walmsley, I. A.; Stroud, C. R., "Dithered-edge sampling of terahertz pulses", Appl. Phys. Lett. 1999, 75, 2181.

(146) Jacobsen, R. H.; Birkelund, K.; Holst, T.; Jepsen, P. U.; Keiding, S. R., "Interpretation of photocurrent correlation measurements used for ultrafast photoconductive switch characterization", J. Appl. Phys. 1996, $79,2649$.

(147) Nemec, H.; Pashkin, A.; Kuzel, P.; Khazan, M.; Schnull, S.; Wilke, I., "Carrier dynamics in low-temperature grown GaAs studied by terahertz emission spectroscopy", J. Appl. Phys. 2001, 90, 1303.

(148) Leitenstorfer, A.; Hunsche, S.; Shah, J.; Nuss, M. C.; Knox, W. H., "Femtosecond charge transport in polar semiconductors", Phys. Rev. Lett. 1999, 82, 5140.

(149) Leitenstorfer, A.; Hunsche, S.; Shah, J.; Nuss, M. C.; Knox, W. H., "Femtosecond high-field transport in compound semiconductors", Phys. Rev. B 2000, 61, 16642.

(150) Markelz, A. G.; Heilweil, E. J., "Temperature-dependent terahertz output from semi-insulating GaAs photoconductive switches", Appl. Phys. Lett. 1998, 72, 2229.

(151) Sekine, N.; Hirakawa, K.; Sogawa, F.; Arakawa, Y.; Usami, N.; Shiraki, Y.; Katoda, T., "Ultrashort lifetime photocarriers in Ge thin films", Appl. Phys. Lett. 1996, 68, 3419.

(152) Nakajima, M.; Takahashi, M.; Hangyo, M., "Temperature dependence of $\mathrm{THz}$ radiation from semi-insulating InP surface", J. Lumines. 2001, 94, 627.

(153) Nakajima, M.; Takahashi, M.; Hangyo, M., "Reversal of subpicosecond carrier transport direction with temperature observed in semi-insulating InP using THz radiation", Physica B 2002, 314, 176.

(154) Xu, L.; Hu, B. B.; Xin, W.; Auston, D. H.; Morse, J. D., "Hot-Electron Dynamics Study by Terahertz Radiation from Large- Aperture Gaas P-IN-Diodes", Appl. Phys. Lett. 1993, 62, 3507.

(155) Kersting, R.; Unterrainer, K.; Strasser, G.; Kauffmann, H. F.; Gornik, E., "Few-cycle THz emission from cold plasma oscillations", Phys. Rev. Lett. 1997, 79, 3038.

(156) Leitenstorfer, A.; Hunsche, S.; Shah, J.; Nuss, M. C.; Knox, W. H., "Ultrafast high-field transport in semiconductors", Physica B 1999, 272, 348.

(157) Yu, P. Y.; Cardona, M. Fundamentals of Semiconductors; 3rd ed.; Springer: New York, 2001

(158) Hirakawa, K.; Wilke, I.; Yamanaka, K.; Roskos, H. G.; Vosseburger, M.; Wolter, F.; Waschke, C.; Kurz, H.; Grayson, M.; Tsui, D. C., "Coherent submillimeter-wave emission from non-equilibrium two- dimensional free carrier plasmas in AlGaAs/GaAs heterojunctions", Surf. Sci. 1996, 362,368 . 
(159) Sekine, N.; Hirakawa, K.; Vossebuerger, M.; Haring-Bolivar, P.; Kurz, H., "Excitation process of two-dimensional plasmons excited by femtosecond laser pulses", Microelectron. Eng. 1999, 47, 289.

(160) Sekine, N.; Yamanaka, K.; Hirakawa, K.; Vosseburger, M.; Haring-Bolivar, P.; Kurz, H., "Observation of terahertz radiation from higher-order two- dimensional plasmon modes in GaAs/AlGaAs single quantum wells", Appl. Phys. Lett. 1999, 74, 1006.

(161) Sekine, N.; Hirakawa, K.; Vosseburger, M.; Haring-Bolivar, P.; Kurz, H. In Compound Semiconductors 1998, 1999.

(162) Planken, P. C. M.; Nuss, M. C.; Brener, I.; Goossen, K. W.; Luo, M. S. C.; Chuang, S. L.; Pfeiffer, L., "Terahertz Emission in Single Quantum-Wells after Coherent Optical-Excitation of Light Hole and Heavy Hole Excitons", Phys. Rev. Lett. 1992, 69, 3800.

(163) Planken, P. C. M.; Nuss, M. C.; Knox, W. H.; Miller, D. A. B.; Goossen, K. W., "Thz Pulses from the Creation of Polarized Electron-Hole Pairs in Biased Quantum-Wells", Appl. Phys. Lett. 1992, 61, 2009.

(164) Planken, P. C. M.; Brener, I.; Nuss, M. C.; Luo, M. S. C.; Chuang, S. L.; Pfeiffer, L. N., "Thz Radiation from Coherent PopulationChanges in Quantum-Wells", Phys. Rev. B 1994, 49, 4668.

(165) Brener, I.; Planken, P. C. M.; Nuss, M. C.; Luo, M. S. C.; Chuang, S. L.; Pfeiffer, L.; Leaird, D. E.; Weiner, A. M., "Coherent Control of Terahertz Emission and Carrier Populations in Semiconductor Heterostructures", J. Opt. Soc. Am. B-Opt. Phys. 1994, 11, 2457.

(166) Sha, W. J.; Rhee, J. K.; Norris, T. B.; Schaff, W. J., "Transient Carrier and Field-Dynamics in Quantum-Well Parallel Transport from the Ballistic to the Quasi-Equilibrium Regime", IEEE $J$. Quantum Electron. 1992, 28, 2445.

(167) Roskos, H. G.; Nuss, M. C.; Shah, J.; Leo, K.; Miller, D. A. B.; Fox, A. M.; Schmittrink, S.; Kohler, K., "Coherent SubmillimeterWave Emission from Charge Oscillations in a Double-Well Potential", Phys. Rev. Lett. 1992, 68, 2216.

(168) Planken, P. C. M.; Brener, I.; Nuss, M. C.; Luo, M. S. C.; Chuang, S. L., "Coherent Control of Terahertz Charge Oscillations in a Coupled- Quantum-Well Using Phase-Locked Optical Pulses", Phys. Rev. B 1993, 48, 4903.

(169) Nuss, M. C.; Planken, P. C. M.; Brener, I.; Roskos, H. G.; Luo, M. S. C.; Chuang, S. L., "Terahertz Electromagnetic-Radiation from Quantum-Wells", Appl. Phys. B-Lasers Opt. 1994, 58, 249.

(170) Luo, M. S. C.; Chuang, S. L.; Planken, P. C. M.; Brener, I.; Roskos, H. G.; Nuss, M. C., "Generation of Terahertz Electromagnetic Pulses from Quantum- Well Structures", IEEE J. Quantum Electron. 1994, 30, 1478.

(171) Sekine, N.; Hirakawa, K.; Arakawa, Y., "Terahertz emission from quantum beats in coupled quantum wells", Jpn. J. Appl. Phys. Part 1 - Regul. Pap. Short Notes Rev. Pap. 1998, 37, 1643.

(172) Morohashi, I.; Komori, K.; Hidaka, T.; Sugaya, T.; Wang, X. L.; Ogura, M.; Nakagawa, T.; Son, C. S., "Terahertz electromagnetic wave generation from quantum nanostructure", Jpn. J. Appl. Phys. Part 1 - Regul. Pap. Short Notes Rev. Pap. 2001, 40, 3012.

(173) Camacho, A. S.; Gutierrez, R. M.; Bohorquez, J., "Mechanism of terahertz emission from an asymmetric double quantum well", Phys. Status Solidi B-Basic Res. 2002, 232, 56.

(174) Morohashi, I.; Komori, K.; Wang, X. L.; Hidaka, T.; Ogura, M.; Watanabe, M., "Excitation wavelength dependence of terahertz electromagnetic wave generation from quantum wire", Jpn. $J$. Appl. Phys. Part 1 - Regul. Pap. Short Notes Rev. Pap. 2002, 41, 2710 .

(175) Zhang, W. M.; Meier, T.; Chernyak, V.; Mukamel, S., "Intraband terahertz emission from coupled semiconductor quantum wells: A model study using the exciton representation", Phys. Rev. B 1999, 60, 2599.

(176) Waschke, C.; Leisching, P.; Bolivar, P. H.; Schwedler, R.; Bruggemann, F.; Roskos, H. G.; Leo, K.; Kurz, H.; Kohler, K., "Detection of Bloch Oscillations in a Semiconductor Superlattice by Time-Resolved Terahertz Spectroscopy and Degenerate 4Wave- Mixing", Solid-State Electron. 1994, 37, 1321.
(177) Waschke, C.; Roskos, H. G.; Leo, K.; Kurz, H.; Kohler, K., "Experimental Realization of the Bloch Oscillator in a Semiconductor Superlattice", Semicond. Sci. Technol. 1994, 9, 416.

(178) Dekorsy, T.; Leisching, P.; Waschke, C.; Kohler, K.; Leo, K.; Roskos, H. G.; Kurz, H., "Terahertz Bloch Oscillations in Semiconductor Superlattices", Semicond. Sci. Technol. 1994, 9, 1959.

(179) Roskos, H. G. In Festkorperprobleme-Advances in Solid State Physics, 1995; Vol. 34.

(180) Dekorsy, T.; Ott, R.; Leisching, P.; Bakker, H. J.; Waschke, C.; Roskos, H. G.; Kurz, H.; Kohler, K., "Time-resolved optical investigations of bloch oscillations in semiconductor superlattices", Solid-State Electron. 1996, 40, 551.

(181) Shimada, Y.; Matsuno, T.; Hirakawa, K., "Terahertz emission due to miniband transport in GaAs/AlGaAs superlattices", Jpn. J. Appl. Phys. Part 1 - Regul. Pap. Short Notes Rev. Pap. 2001, 40, 3009.

(182) Shimada, Y.; Hirakawa, K.; Lee, S. W., "Time-resolved terahertz emission spectroscopy of wide miniband GaAs/AlGaAs superlattices", Appl. Phys. Lett. 2002, 81, 1642.

(183) Sudzius, M.; Lyssenko, V. G.; Loser, F.; Leo, K.; Dignam, M. M.; Kohler, K., "Optical control of Bloch-oscillation amplitudes: From harmonic spatial motion to breathing modes", Phys. Rev. B 1998, 57, R12693.

(184) Shimada, Y.; Hirakawa, K. In Compound Semiconductors 2001, 2002.

(185) Hokomoto, Y.; Kadoya, Y.; Yamanishi, M., "THz electromagnetic wave radiation from coherent oscillation of exciton population in high-Q semiconductor microcavities", Appl. Phys. Lett. 1999, 74, 3839.

(186) Sakurada, T.; Kadoya, Y.; Yamanishi, M., "THz electromagnetic wave radiation from bulk semiconductor microcavities excited by short laser pulses", Jpn. J. Appl. Phys. Part 2 - Lett. 2002, 41, L256.

(187) Walecki, W. J.; Some, D.; Kozlov, V. G.; Nurmikko, A. V., "Terahertz Electromagnetic Transients as Probes of a 2-Dimensional Electron-Gas", Appl. Phys. Lett. 1993, 63, 1809.

(188) Some, D.; Nurmikko, A. V., "Real-Time Electron-Cyclotron Oscillations Observed by Terahertz Techniques in Semiconductor Heterostructures", Appl. Phys. Lett. 1994, 65, 3377.

(189) Kersting, R.; Bratschitsch, R.; Strasser, G.; Unterrainer, K.; Heyman, J. N., "Sampling a terahertz dipole transition with subcycle time resolution", Opt. Lett. 2000, 25, 272.

(190) Ascazubi, R.; Akin, O. C.; Zaman, T.; Kersting, R.; Strasser, G., "Dephasing in modulation-doped quantum structures probed by $\mathrm{THz}$ time-domain spectroscopy", Appl. Phys. Lett. 2002, 81, 4344.

(191) Tonouchi, M.; Tani, M.; Wang, Z.; Sakai, K.; Tomozawa, S.; Hangyo, M.; Murakami, Y.; Nakashima, S. I., "Ultrashort electromagnetic pulse radiation from YBCO thin films excited by femtosecond optical pulse", Jpn. J. Appl. Phys. Part 1 - Regul. Pap. Short Notes Rev. Pap. 1996, 35, 2624.

(192) Tonouchi, M.; Tani, M.; Wang, Z.; Sakai, K.; Wada, N.; Hangyo, M., "Terahertz emission study of femtosecond time-transient nonequilibrium state in optically excited YBa2Cu3O7-delta thin films", Jpn. J. Appl. Phys. Part 2 - Lett. 1996, 35, L1578.

(193) Hangyo, M.; Tomozawa, S.; Murakami, Y.; Tonouchi, M.; Tani, M.; Wang, Z.; Sakai, K.; Nakashima, S., "Terahertz radiation from superconducting $\mathrm{YBa} 2 \mathrm{Cu} 3 \mathrm{O} 7-$ delta thin films excited by femtosecond optical pulses", Appl. Phys. Lett. 1996, 69, 2122.

(194) Jaekel, C.; Roskos, H. G.; Kurz, H., "Emission of picosecond electromagnetic pulses from optically excited superconducting bridges", Phys. Rev. B 1996, 54, R6889.

(195) Jaekel, C.; Roskos, H. G.; Kurz, H., "Ultrafast optoelectronic switches based on high-T-c superconductors", IEEE Trans. Appl. Supercond. 1997, 7, 3722.

(196) Tani, M.; Tonouchi, M.; Wang, Z.; Sakai, K.; Hangyo, M.; Tomozawa, S.; Murakami, Y., "Enhanced sub-picosecond electromagnetic radiation from YBa2Cu3O7-delta thin-film bow-tie antennas excited with femtoseconds laser pulses", Jpn. J. Appl. Phys. Part 2 - Lett. 1996, 35, L1184.

(197) Tonouchi, M.; Tani, M.; Wang, Z.; Sakai, K.; Wada, N.; Hangyo, M., "Novel terahertz radiation from flux-trapped $\mathrm{YBa} 2 \mathrm{Cu} 3 \mathrm{O} 7-$ delta thin films excited by femtosecond laser pulses", Jpn. J. Appl. Phys. Part 2 Lett. 1997, 36, L93. 
(198) Tonouchi, M.; Wada, N.; Shikii, S.; Hangyo, M.; Tani, M.; Sakai, K., "Terahertz emission properties from flux-trapped YBCO thin films", Physica C 1997, 293, 82.

(199) Hangyo, M.; Wada, N.; Tonouchi, M.; Tani, M.; Sakai, K., "Ultrafast optical response and terahertz radiation from high-T- $\mathrm{C}$ superconductor", IEICE Trans. Electron. 1997, E80C, 1282.

(200) Hangyo, M.; Tomozawa, S.; Murakami, Y.; Tonouchi, M.; Tani, M.; Wang, Z.; Sakai, K., "Ultrafast photoexcited quasiparticle dynamics in YBCO films observed by THz emission", IEEE Trans. Appl. Supercond. 1997, 7, 2909.

(201) Hangyo, M.; Tomozawa, S.; Murakami, Y.; Tonouchi, M.; Tani, M.; Wang, Z.; Sakai, K., "THz emission from high-T-c superconductors by optical pulse excitation", IEEE Trans. Appl. Supercond. 1997, 7, 3730.

(202) Iguchi, I.; Kume, E.; Takahashi, H., "Emitted spectra of electromagnetic waves from a tunnel-injected nonequilibrium highT-c YBa2Cu3O7-y superconductor", Phys. Rev. B 2000, 62, 5370.

(203) Wald, H.; Steigmeier, C.; Seidel, P.; Nashima, S.; Tonouchi, M.; Hangyo, M., "Comparison of the electromagnetic pulse emission from $\mathrm{YBa} 2 \mathrm{Cu} 3 \mathrm{O} 7$-delta and $\mathrm{Y} 0.7 \mathrm{Pr} 0.3 \mathrm{Ba} 2 \mathrm{Cu} 3 \mathrm{O} 7$ excited by femtosecond laser pulses", Physica C 2000, 341, 1899.

(204) Yoshimura, T.; Kiwa, T.; Tonouchi, M., "Terahertz emission from YBa2Cu3O7-delta Josephson junctions excited by femtosecond laser", Physica C 2001, 362, 329.

(205) Wald, H.; Seidel, P.; Tonouchi, M., "Pump-and-probe terahertz method to investigate $\mathrm{YBa} 2 \mathrm{Cu} 3 \mathrm{O} 7$-delta thin films", Physica $C$ 2002, 367, 308

(206) Wald, H.; Seidel, P.; Tonouchi, M., "Carrier dynamics in YBa2Cu3O7-x studied by pump and probe terahertz excitation", Physica C 2001, 362, 324.

(207) Wald, H.; Seidel, P.; Tonouchi, M., "Pump and probe terahertz beam excitation technique to investigate carrier dynamics in YBa2Cu3O7-delta thin films", Physica C 2001, 357, 146.

(208) Wald, H.; Schmidt, F.; Seidel, P.; Tonouchi, M., "Terahertz radiation emitted by dipole antennas of $\mathrm{YBa} 2 \mathrm{Cu} 3 \mathrm{O} 7-$ delta/PrBa2Cu3O7 multilayers", Supercond. Sci. Technol. 2002, 15,1494

(209) Tominari, Y.; Kiwa, T.; Murakami, H.; Tonouchi, M.; Wald, H.; Seidel, P.; Schneidewind, H., "Resonant terahertz radiation from Tl2Ba2CaCu2O8+delta thin films by ultrafast optical pulse excitation", Appl. Phys. Lett. 2002, 80, 3147.

(210) Tominari, Y.; Kiwa, T.; Murakami, H.; Tonouchi, M.; Wald, H.; Seidel, P.; Schneidewind, H., "Coherent terahertz radiation from $\mathrm{T} 2 \mathrm{Ba} 2 \mathrm{CaCu} 2 \mathrm{O} 8+$ delta thin films excited by optical laser pulse under magnetic field", Physica C 2003, 388, 481.

(211) Beard, M. C.; Turner, G. M.; Schmuttenmaer, C. A., "Measurement of electromagnetic radiation emitted during rapid intramolecular electron transfer", J. Am. Chem. Soc. 2000, 122, 11541.

(212) Beard, M. C.; Turner, G. M.; Schmuttenmaer, C. A., "Measuring intramolecular charge transfer via coherent generation of $\mathrm{THz}$ radiation", J. Phys. Chem. A 2002, 106, 878

(213) Groot, M. L.; Vos, M. H.; Schlichting, I.; van Mourik, F.; Joffre, M.; Lambry, J. C.; Martin, J. L., "Coherent infrared emission from myoglobin crystals: An electric field measurement", Proc. Natl. Acad. Sci. U. S. A. 2002, 99, 1323.

(214) Kida, N.; Tonouchi, M., "Terahertz radiation from magnetoresistive Pr0.7Ca0.3MnO3 thin films", Appl. Phys. Lett. 2001, 78, 4115 .

(215) Kida, N.; Tonouchi, M., "Reversible and bistable terahertz radiation from magnetoresistive $\mathrm{Pr} 0.7 \mathrm{Ca} 0.3 \mathrm{MnO} 3$ thin films", Appl. Phys. Lett. 2003, 82, 3412.

(216) Wilke, I.; MacLeod, A. M.; Gillespie, W. A.; Berden, G.; Knippels, G. M. H.; van der Meer, A. F. G., "Real-time single-shot electron bunch length measurements", Nucl. Instrum. Methods Phys. Res. Sect. A-Accel. Spectrom. Dect. Assoc. Equip. 2002, 483, 282.

(217) Wilke, I.; MacLeod, A. M.; Gillespie, W. A.; Berden, G.; Knippels, G. M. H.; van der Meer, A. F. G., "Single-shot electron-beam bunch length measurements", Phys. Rev. Lett. 2002, 88, 124801.
(218) Pedersen, J. E.; Lyssenko, V. G.; Hvam, J. M.; Jepsen, P. U.; Keiding, S. R.; Sorensen, C. B.; Lindelof, P. E., "Ultrafast Local Field-Dynamics in Photoconductive Thz Antennas", Appl. Phys. Lett. 1993, 62, 1265.

(219) Brorson, S. D.; Zhang, J. C.; Keiding, S. R., "Ultrafast Carrier Trapping and Slow Recombination in Ion- Bombarded Silicon-on-Sapphire Measured Via Thz Spectroscopy", Appl. Phys. Lett. 1994, 64, 2385.

(220) Hu, B. B.; Desouza, E. A.; Knox, W. H.; Cunningham, J. E.; Nuss, M. C.; Kuznetsov, A. V.; Chuang, S. L., "Identifying the Distinct Phases of Carrier Transport in Semiconductors with 10-Fs Resolution", Phys. Rev. Lett. 1995, 74, 1689.

(221) Taylor, A. J.; Rodriguez, G.; Some, D., "Ultrafast field dynamics in large-aperture photoconductors", Opt. Lett. 1997, 22, 715.

(222) Huggard, P. G.; Cluff, J. A.; Shaw, C. J.; Andrews, S. R.; Linfield, E. H.; Ritchie, D. A., "Coherent control of cyclotron emission from a semiconductor using sub-picosecond electric field transients", Appl. Phys. Lett. 1997, 71, 2647.

(223) Kersting, R.; Heyman, J. N.; Strasser, G.; Unterrainer, K., "Coherent plasmons in n-doped GaAs", Phys. Rev. B 1998, 58, 4553.

(224) Tonouchi, M.; Kawasaki, N.; Yoshimura, T.; Wald, H.; Seidel, P., "Pump and probe terahertz generation study of ultrafast carrier dynamics in low-temperature grown-GaAs", Jpn. J. Appl. Phys. Part 2 - Lett. 2002, 41, L706.

(225) Weling, A. S.; Hu, B. B.; Froberg, N. M.; Auston, D. H., "Generation of Tunable Narrow-Band Thz Radiation from Large- Aperture Photoconducting Antennas", Appl. Phys. Lett. 1994, 64, 137.

(226) Weling, A. S.; Auston, D. H., "Novel sources and detectors for coherent tunable narrow-band terahertz radiation in free space", J. Opt. Soc. Am. B-Opt. Phys. 1996, 13, 2783.

(227) Liu, Y. Q.; Park, S. G.; Weiner, A. M., "Enhancement of narrow-band terahertz radiation from photoconducting antennas by optical pulse shaping", Opt. Lett. 1996, 21, 1762.

(228) Siders, C. W.; Siders, J. L. W.; Taylor, A. J.; Park, S. G.; Melloch, M. R.; Weiner, A. M., "Generation and characterization of terahertz pulse trains from biased, large-aperture photoconductors", Opt. Lett. 1999, 24, 241.

(229) Sohn, J. Y.; Ahn, Y. H.; Park, D. J.; Oh, E.; Kim, D. S., "Tunable terahertz generation using femtosecond pulse shaping", Appl. Phys. Lett. 2002, 81,13 .

(230) Haran, G.; Sun, W. D.; Wynne, K.; Hochstrasser, R. M., "Femtosecond far-infrared pump-probe spectroscopy: A new tool for studying lowfrequency vibrational dynamics in molecular condensed phases", Chem. Phys. Lett. 1997, 274, 365.

(231) McElroy, R.; Wynne, K., "Ultrafast dipole solvation measured in the far infrared", Phys. Rev. Lett. 1997, 79, 3078.

(232) Greene, B. I.; Federici, J. F.; Dykaar, D. R.; Levi, A. F. J.; Pfeiffer, L., "Picosecond Pump and Probe Spectroscopy Utilizing Freely Propagating Terahertz Radiation", Opt. Lett. 1991, 16, 48.

(233) Saeta, P. N.; Federici, J. F.; Greene, B. I.; Dykaar, D. R., "Intervalley Scattering in Gaas and Inp Probed by Pulsed Far- Infrared Transmission Spectroscopy", Appl. Phys. Lett. 1992, 60, 1477.

(234) Chen, Y.; Prabhu, S. S.; Ralph, S. E.; McInturff, D. T., "Trapping and recombination dynamics of low-temperature-grown InGaAs/InAlAs multiple quantum wells", Appl. Phys. Lett. 1998, 72, 439.

(235) Messner, C.; Kostner, H.; Hopfel, R. A.; Unterrainer, K., "Time-resolved THz spectroscopy of proton-bombarded InP", J. Opt. Soc. Am. B-Opt. Phys. 2001, 18, 1369.

(236) Lui, K. P. H.; Hegmann, F. A., "Ultrafast carrier relaxation in radiationdamaged silicon on sapphire studied by optical-pump-terahertz-probe experiments", Appl. Phys. Lett. 2001, 78, 3478.

(237) Lui, K. P. H.; Hegmann, F. A., "Fluence- and temperature-dependent studies of carrier dynamics in radiation-damaged silicon-on-sapphire and amorphous silicon", J. Appl. Phys. 2003, 93, 9012.

(238) Jepsen, P. U.; Schairer, W.; Libon, I. H.; Lemmer, U.; Hecker, N. E.; Birkholz, M.; Lips, K.; Schall, M., "Ultrafast carrier trapping in microcrystalline silicon observed in optical pump-terahertz probe measurements", Appl. Phys. Lett. 2001, 79, 1291.

(239) Yarotski, D. A.; Averitt, R. D.; Negre, N.; Crooker, S. A.; Taylor, A. J.; Donati, G. P.; Stintz, A.; Lester, L. F.; Malloy, K. J., "Ultrafast carrier- 
relaxation dynamics in self-assembled InAs/GaAs quantum dots", J. Opt. Soc. Am. B-Opt. Phys. 2002, 19, 1480.

(240) Segschneider, G.; Jacob, F.; Loffler, T.; Roskos, H. G.; Tautz, S.; Kiesel, P.; Dohler, G., "Free-carrier dynamics in low-temperaturegrown GaAs at high excitation densities investigated by timedomain terahertz spectroscopy", Phys. Rev. B 2002, 65, 125205.

(241) Hegmann, F. A.; Tykwinski, R. R.; Lui, K. P. H.; Bullock, J. E.; Anthony, J. E., "Picosecond transient photoconductivity in functionalized pentacene molecular crystals probed by terahertz pulse spectroscopy", Phys. Rev. Lett. 2002, 89, 227403.

(242) Stanton, C. J.; Bailey, D. W., "Rate-Equations For the Study of Femtosecond Intervalley Scattering in Compound Semiconductors", Phys. Rev. B 1992, 45, 8369.

(243) Federici, J. F.; Greene, B. I.; Saeta, P. N.; Dykaar, D. R.; Sharifi, F.; Dynes, R. C., "Direct Picosecond Measurement of Photoinduced Cooper-Pair Breaking in Lead", Phys. Rev. B 1992, 46, 11153.

(244) Groeneveld, R. H. M.; Grischkowsky, D., "Picosecond TimeResolved Far-Infrared Experiments on Carriers and Excitons in Gaas-Algaas Multiple-Quantum Wells", J. Opt. Soc. Am. B-Opt. Phys. 1994, 11, 2502.

(245) Flanders, B. N.; Arnett, D. C.; Scherer, N. F., "Optical pumpterahertz probe spectroscopy utilizing a cavity- dumped oscillatordriven terahertz spectrometer", IEEE J. Sel. Top. Quantum Electron. 1998, 4, 353.

(246) Schall, M.; Jepsen, P. U., "Photoexcited GaAs surfaces studied by transient terahertz time- domain spectroscopy", Opt. Lett. 2000, 25, 13.

(247) Knoesel, E.; Bonn, M.; Shan, J.; Heinz, T. F., "Charge transport and carrier dynamics in liquids probed by $\mathrm{THz}$ time-domain spectroscopy", Phys. Rev. Lett. 2001, 86, 340.

(248) Jamison, S. P.; Shen, J. L.; Jones, D. R.; Issac, R. C.; Ersfeld, B.; Clark, D.; Jaroszynski, D. A., "Plasma characterization with terahertz time-domain measurements", J. Appl. Phys. 2003, 93, 4334.

(249) Nuss, M. C.; Auston, D. H.; Capasso, F., "Direct Subpicosecond Measurement of Carrier Mobility of Photoexcited Electrons in Gallium-Arsenide", Phys. Rev. Lett. 1987, 58, 2355.

(250) Zielbauer, J.; Wegener, M., "Ultrafast optical pump THz-probe spectroscopy on silicon", Appl. Phys. Lett. 1996, 68, 1223.

(251) Huber, R.; Tauser, F.; Brodschelm, A.; Bichler, M.; Abstreiter, G.; Leitenstorfer, A., "How many-particle interactions develop after ultrafast excitation of an electron-hole plasma", Nature 2001, 414, 286.

(252) Huber, R.; Tauser, F.; Brodschelm, A.; Bichler, M.; Abstreiter, G.; Leitenstorfer, A., "Femtosecond buildup of Coulomb screening in photoexcited GaAs probed via ultrabroadband $\mathrm{THz}$ spectroscopy", J. Lumines. 2001, 94, 555.

(253) Huber, R.; Tauser, F.; Brodschelm, A.; Leitenstorfer, A., "Femtosecond buildup of a many-body resonance observed via two- dimensional THz time-domain spectroscopy", Phys. Status Solidi B-Basic Res. 2002, 234, 207.

(254) Leitenstorfer, A.; Huber, R.; Tauser, F.; Brodschelm, A.; Bichler, M.; Abstreiter, G., "Femtosecond buildup of Coulomb screening in a photoexcited electron-hole plasma", Physica B 2002, 314, 248.

(255) Kaindl, R. A.; Carnahan, M. A.; Hagele, D.; Lovenich, R.; Chemla, D. S., "Ultrafast terahertz probes of transient conducting and insulating phases in an electron-hole gas", Nature 2003, 423, 734.

(256) Averitt, R. D.; Rodriguez, G.; Siders, J. L. W.; Trugman, S. A.; Taylor, A. J., "Conductivity artifacts in optical-pump THz-probe measurements of YBa2Cu3O7", J. Opt. Soc. Am. B-Opt. Phys. 2000, 17, 327.

(257) Averitt, R. D.; Rodriguez, G.; Lobad, A. I.; Siders, J. L. W.; Trugman, S. A.; Taylor, A. J., "Nonequilibrium superconductivity and quasiparticle dynamics in YBa2Cu3O7-delta", Phys. Rev. B 2001, 63, 140502.

(258) Brorson, S. D.; Buhleier, R.; Trofimov, I. E.; White, J. O.; Ludwig, C.; Balakirev, F. F.; Habermeier, H. U.; Kuhl, J., "Electrodynamics of high-temperature superconductors investigated with coherent terahertz pulse spectroscopy", J. Opt. Soc. Am. B-Opt. Phys. 1996, 13, 1979.

(259) Averitt, R. D.; Lobad, A. I.; Kwon, C.; Trugman, S. A.; Thorsmolle, V. K.; Taylor, A. J., "Ultrafast conductivity dynamics in colossal magnetoresistance manganites", Phys. Rev. Lett. 2001, 87, 017401.

(260) Beard, M. C.; Schmuttenmaer, C. A., "Using the finite-difference timedomain pulse propagation method to simulate time-resolved $\mathrm{THz}$ experiments", J. Chem. Phys. 2001, 114, 2903.

(261) Beard, M. C.; Turner, G. M.; Schmuttenmaer, C. A. In Liquid Dynamics: Experiment, Simulation, and Theory, 2002; Vol. 820.

(262) Beard, M. C.; Turner, G. M.; Schmuttenmaer, C. A., "Size-dependent photoconductivity in CdSe nanoparticles as measured by time-resolved terahertz spectroscopy", Nano Lett. 2002, 2, 983.

(263) Turner, G. M.; Beard, M. C.; Schmuttenmaer, C. A., "Carrier localization and cooling in dye-sensitized nanocrystalline titanium dioxide", J. Phys. Chem. B 2002, 106, 11716.

(264) Smith, N. V., "Classical generalization of the Drude formula for the optical conductivity", Phys. Rev. B 2001, 64, 155106.

(265) Cook, D. J.; Chen, J. X.; Morlino, E. A.; Hochstrasser, R. M., "Terahertz-field-induced second-harmonic generation measurements of liquid dynamics", Chem. Phys. Lett. 1999, 309, 221.

(266) Ahn, J.; Hutchinson, D. N.; Rangan, C.; Bucksbaum, P. H., "Quantum phase retrieval of a Rydberg wave packet using a half- cycle pulse", Phys. Rev. Lett. 2001, 86, 1179.

(267) Ahn, J.; Rangan, C.; Hutchinson, D. N.; Bucksbaum, P. H., "Quantumstate information retrieval in a Rydberg-atom data register", Phys. Rev. A 2002, 66, 022312.

(268) Jones, R. R.; You, D.; Bucksbaum, P. H., "Ionization of Rydberg Atoms by Subpicosecond Half-Cycle Electromagnetic Pulses", Phys. Rev. Lett. 1993, 70, 1236

(269) Jones, R. R.; Tielking, N. E.; You, D.; Raman, C.; Bucksbaum, P. H., "Ionization of Oriented Rydberg States by Subpicosecond Half- Cycle Electromagnetic Pulses", Phys. Rev. A 1995, 51, R2687.

(270) Raman, C.; Conover, C. W. S.; Sukenik, C. I.; Bucksbaum, P. H., "Ionization of Rydberg wave packets by subpicosecond, half-cycle electromagnetic pulses", Phys. Rev. Lett. 1996, 76, 2436.

(271) Raman, C.; Weinacht, T. C.; Bucksbaum, P. H., "Stark wave packets viewed with half-cycle pulses", Phys. Rev. A 1997, 55, R3995.

(272) Rangan, C.; Bucksbaum, P. H., "Optimally shaped terahertz pulses for phase retrieval in a Rydberg-atom data register", Phys. Rev. A 2001, 64, 033417.

(273) Rangan, C.; Ahn, J.; Hutchinson, D. N.; Bucksbaum, P. H., "Control of Rydberg atoms to perform Grover's search algorithm", J. Mod. Opt. 2002, 49, 2339.

(274) Reinhold, C. O.; Burgdorfer, J.; Jones, R. R.; Raman, C.; Bucksbaum, P. H., "Scaled-Time Dynamics of Ionization of Rydberg Stark States by Half-Cycle Pulses", J. Phys. B-At. Mol. Opt. Phys. 1995, 28, L457.

(275) Manescu, C.; Krause, J. L.; Schafer, K. J., "Ionization of Stark states with half-cycle pulses: Interference effects in the continuum", Phys. Rev. A 2003, 68 .

(276) Hache, A.; Kostoulas, Y.; Atanasov, R.; Hughes, J. L. P.; Sipe, J. E.; vanDriel, H. M., "Observation of coherently controlled photocurrent in unbiased, bulk GaAs", Appl. Phys. Lett. 1997, 78, 306.

(277) Kral, P.; Sipe, J. E., "Quantum kinetic theory of two-beam current injection in bulk semiconductors", Phys. Rev. B 2000, 61, 5381.

(278) Cote, D.; Fraser, J. M.; DeCamp, M.; Bucksbaum, P. H.; van Driel, H. M., "THz emission from coherently controlled photocurrents in GaAs", Appl. Phys. Lett. 1999, 75, 3959.

(279) Cote, D.; Laman, N.; van Driel, H. M., "Rectification and shift currents in GaAs", Appl. Phys. Lett. 2002, 80, 905.

(280) Jepsen, P. U.; Keiding, S. R., "Radiation-Patterns from Lens-Coupled Terahertz Antennas", Opt. Lett. 1995, 20, 807.

(281) Joshi, R. P.; Dharamsi, A. N., "Effect of Coherent ElectromagneticRadiation on the Ultrafast Electron Dynamics of Laser-Excited Bulk Photoconductors", J. Appl. Phys. 1993, 74, 3215.

(282) Khurgin, J. B., "Optical Rectification and Terahertz Emission in Semiconductors Excited above the Band-Gap", J. Opt. Soc. Am. B-Opt. Phys. 1994, 11, 2492. 
(283) Abe, M.; Madhavi, S.; Shimada, Y.; Otsuka, Y.; Hirakawa, K.; Tomizawa, K., "Transient carrier velocities in bulk GaAs: Quantitative comparison between terahertz data and ensemble Monte Carlo calculations", Appl. Phys. Lett. 2002, 81, 679.

(284) Piao, Z. S.; Tani, M.; Sakai, K., "Carrier dynamics and terahertz radiation in photoconductive antennas", Jpn. J. Appl. Phys. Part 1 Regul. Pap. Short Notes Rev. Pap. 2000, 39, 96.

(285) You, D.; Bucksbaum, P. H., "Propagation of half-cycle far infrared pulses", J. Opt. Soc. Am. B-Opt. Phys. 1997, 14, 1651.

(286) Kuzel, P.; Khazan, M. A.; Kroupa, J., "Spatiotemporal transformations of ultrashort terahertz pulses", J. Opt. Soc. Am. BOpt. Phys. 1999, 16, 1795.

(287) Nekkanti, S.; Sullivan, D.; Citrin, D. S., "Simulation of spatiotemporal terahertz pulse shaping in 3-D using conductive apertures of finite thickness", IEEE J. Quantum Electron. 2001, 37, 1226.

(288) Nemec, H.; Kadlec, F.; Kuzel, P., "Methodology of an optical pump-terahertz probe experiment: An analytical frequency-domain approach", J. Chem. Phys. 2002, 117, 8454.

(289) Cote, D.; Sipe, J. E.; van Driel, H. M., "Simple method for calculating the propagation of terahertz radiation in experimental geometries", J. Opt. Soc. Am. B-Opt. Phys. 2003, 20, 1374.

(290) Lachaine, J. M.; Hawton, M.; Sipe, J. E.; Dignam, M. M., "Asymmetry in the excitonic Wannier-Stark ladder: A mechanism for the stimulated emission of terahertz radiation", Phys. Rev. B 2000, 62, R4829.

(291) Okumura, K.; Tanimura, Y., "Two-dimensional THz spectroscopy of liquids: non-linear vibrational response to a series of $\mathrm{THz}$ laser pulses", Chem. Phys. Lett. 1998, 295, 298.

(292) Kindt, J. T.; Schmuttenmaer, C. A., "Theory for determination of the low-frequency time-dependent response function in liquids using time-resolved terahertz pulse spectroscopy", J. Chem. Phys. 1999, 110, 8589 .

(293) Feise, M. W.; Citrin, D. S., "Semiclassical theory of terahertz multiple-harmonic generation in semiconductor superlattices", Appl. Phys. Lett. 1999, 75, 3536.

(294) Asplund, M. C.; Zanni, M. T.; Hochstrasser, R. M., "Twodimensional infrared spectroscopy of peptides by phase- controlled femtosecond vibrational photon echoes", Proc. Natl. Acad. Sci. U. S. A. 2000, 97, 8219 .

(295) Golonzka, O.; Khalil, M.; Demirdoven, N.; Tokmakoff, A., "Vibrational anharmonicities revealed by coherent twodimensional infrared spectroscopy", Phys. Rev. Lett. 2001, 86, 2154.

(296) Hybl, J. D.; Albrecht, A. W.; Faeder, S. M. G.; Jonas, D. M., "Two-dimensional electronic spectroscopy", Chem. Phys. Lett. 1998, 297, 307.

(297) Cook, D.J., Personal communication, 2003.

(298) Dlott, D. D., "Optical Phonon Dynamics in Molecular-Crystals", Annu. Rev. Phys. Chem. 1986, 37, 157.

(299) Beard, M. C.; Turner, G. M.; Murphy, J. E.; Micic, O. I.; Hanna, M. C.; Nozik, A. J.; Schmuttenmaer, C. A., "Electronic coupling in InP nanoparticle arrays", Nano Lett. 2003, 3, 1695.

(300) Service, R. F., "Molecular electronics - Next-generation technology hits an early midlife crisis", Science 2003, 302, 556.

(301) Beaurepaire, E.; Turner, G. M.; Harrel, S. M.; Beard, M. C.; Bigot, J.-Y.; Schmuttenmaer, C. A., "Coherent $\mathrm{THz}$ Emission from Ferromagnetic Films Excited by Visible Femtosecond Laser Pulses", Appl. Phys. Lett. 2003, Submitted. 